\title{
Single-Cell Expression Analyses of Embryonic Cardiac Progenitor Cells
}

\author{
Kenta Yashiro and Ken Suzuki
}

\begin{abstract}
For this decade, heart development has been extensively elucidated by the introduction of the concepts of "heart fields" and "cardiac progenitor cells (CPCs)". It is believed that multipotent CPCs are specified among the most anterior part of lateral plate mesoderm as belonging to the two anatomical fields; the first heart field (FHF), which is the future left ventricle and atria, and the second heart field (SHF), which is the future right ventricle and outflow tract of the heart. However, the paradigm of two heart fields dependent on conventional marker genes is still disputed, so the existence of independent CPCs specific to each HF remains an open question. In addition, the molecular mechanism underlying the specification of CPCs remains largely unknown. A single-cell transcriptomic approach, which is realized by the recent advances in molecular biology, can be one of the solutions to bring some breakthrough in this subject.

\section{Keywords}

Cardiac progenitor cells • First heart field • Second heart field • Single-cell transcriptomics
\end{abstract}

\subsection{Introduction}

Better understanding of the molecular mechanism of heart development is vital to clarify the pathophysiology of congenital heart diseases and will benefit us in terms of prediction, prevention and treatment of such diseases in the future. In addition,

K. Yashiro $(\bowtie) \cdot K$. Suzuki

Translational Medicine and Therapeutics, William Harvey Research Institute, Barts and The London School of Medicine and Dentistry, Queen Mary University of London, Charterhouse Square, London EC1M 6BQ, UK

e-mail: k.yashiro@qmul.ac.uk

T. Nakanishi et al. (eds.), Etiology and Morphogenesis of Congenital Heart Disease, DOI 10.1007/978-4-431-54628-3_10 
the information of multipotent cardiac progenitor cells (CPCs) is expected to be valuable for stem cell-based regeneration therapy for the failed heart. However, our knowledge is still insufficient for full translation to the clinical arena.

Here, we review the current knowledge of the earliest phase of cardiac development in mice focusing on CPC specification and discuss the potential of single-cell transcriptomic analyses to elucidate the mechanism $[1,2]$.

\subsection{CPCs of the Two Heart Fields}

The heart is one of the first anatomical structures formed during embryogenesis. Fate mapping studies indicated that the nascent mesodermal cells contributing to the heart came through the anterior half of primitive streak on embryonic day (E) 6.5 (Fig. 10.1a) [3]. These mesodermal cells migrate anteriorly acquiring the identity of lateral plate mesoderm. At E7.5, the anterior part of lateral plate mesoderm is identified as the heart fields, the direct source of the heart tube $[1,4]$. Then the subsequent morphogenesis to create the heart with the four chambers, including the heart tube formation and looping, occurs.

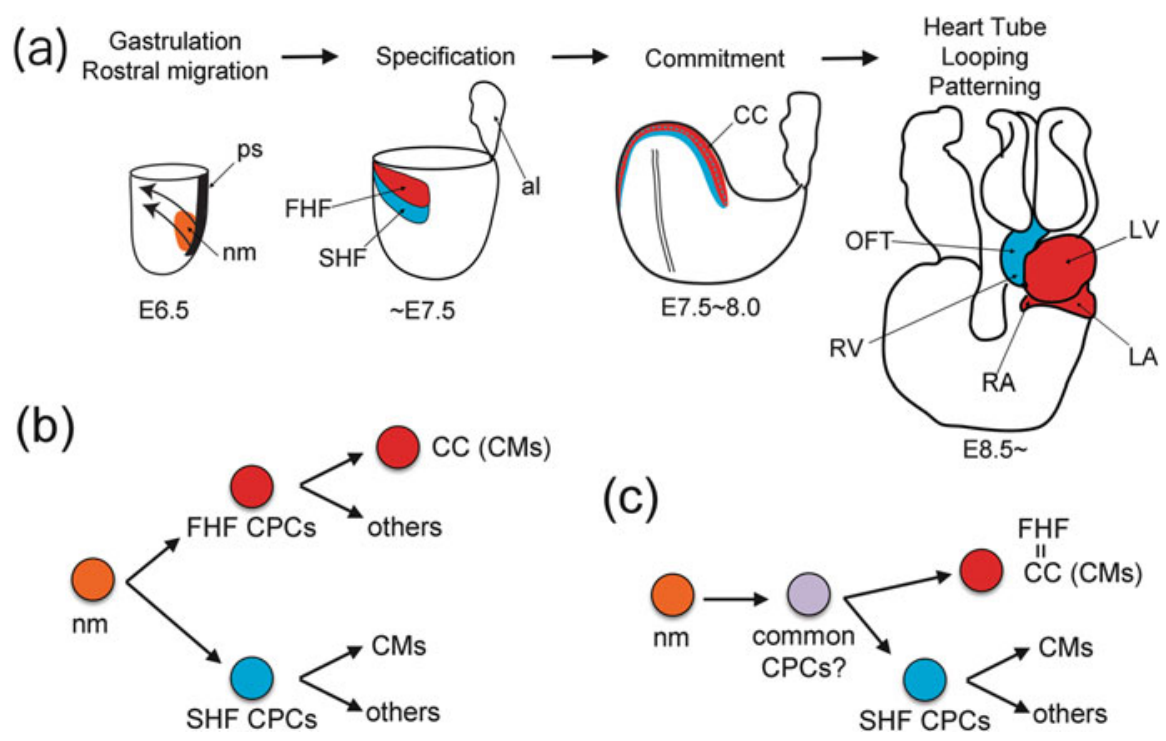

Fig. 10.1 The heart development. (a) Schematic illustration of the heart development. The progeny of FHF is indicated by red and that of SHF is by blue. (b) A hypothetical model of the lineage tree of the heart mesoderm. In this model, it is hypothesized that the independent multipotent CPCs of each heart field. (c) An alternative model of the heart mesoderm lineage tree. In this model, common CPCs are hypothesized, and only in the case of SHF, the multipotent state might be maintained. al allantois, $C C$ cardiac crescent, $C M s$ cardiomyocytes, $L A$ left atrium, $L V$ left ventricle, $n m$ nascent mesoderm, $O F T$ outflow tract, $p s$ primitive streak, $R A$ right atrium, $R V$ right ventricle 
According to the fate map, the anatomical heart fields are divided into two major subgroups, the first heart field (FHF) and the second heart field (SHF) (Fig. 10.1a) [1]. FHF is the classical cardiac crescent and is known to contribute to the left ventricle and parts of the atria. SHF contributes to the right ventricle, outflow tract and also parts of atria. These anatomical fields are currently distinguished with the following molecular markers. The genes encoding transcription factors, $N k x 2-5$ and Tbx5, mark the FHF, whereas Isll marks the SHF at E7.5 in the mouse [1].

CPCs are believed to appear in the period from gastrulation (E6.5) to the heart fields' formation (E7.5). It is thought that CPCs have been already committed toward the heart but are multipotent to give rise to cardiomyocytes, electric conduction system, smooth muscle and endothelium (Fig. 10.1b, c) [1]. Given the paradigm of the anatomical two heart fields, CPCs are classified into FHF CPCs and SHF CPCs (Fig. 10.1b). Unfortunately multipotent FHF CPCs have not been identified (Fig. 10.1c). The cells in the cardiac crescent, the theoretical progeny of FHF CPCs, are unlikely to be "multipotent progenitors", because these already express the terminal differentiation markers of cardiomyocytes, such as Actcl and Myl7 [1, 5, 6]. To add to this, no marker is available to identify FHF before $N k x 2-5$ and $T b x 5$ expression. By contrast, the presence of multipotent SHF CPCs, which are marked by Isll expression, seems to be validated with clonal tracing experiments [7]. However, the paradigm of FHF and SHF based on Nkx2-5 and Isll is now disputed [1]. The expression of Isll was shown to be not specific for SHF and was suggested to represent only the developmental stages [8, 9]. Thus, true characteristics of CPCs should be clarified with revalidation of the conventional biomarkers and the concept of two heart fields.

\subsection{CPC Specification}

The molecular mechanism underlying the specification of CPCs remains largely unknown. In the mouse embryo, specification might occur inside the heart fields $[1,4]$. When the nascent mesoderm cells at E6.5 containing the presumptive heart fields were transplanted heterotopically to a location other than the heart fields, the fate was not the heart but the same as the cells of the recipient surrounding the graft [4]. On the other hand, when the same graft was transplanted to the heart fields of E7.5, it was committed almost only to the heart. These suggest that the migrating presumptive heart fields are plastic and that some inductive cue for cardiac specification is present within the heart fields. The surrounding tissue, especially the endoderm, likely provides such an inductive cue [1, 4]. However, we cannot exclude the possibility that some sequential events of specification take place during the gastrulation and migration so that the unfixed cardiac competence might be gradually and sequentially consolidated.

It has been shown that the signalling pathways of WNT, FGF, BMP, NODAL (ACTIVIN), SHH and NOTCH are involved in the specification $[1,10]$. However, validating the precise role of each signalling pathway is difficult because their 
accurate cardiogenic function has been hard to distinguish from their overall effects. Thus, some alternative strategy is needed to bring a breakthrough.

\subsection{The Potential of Single-Cell Transcriptomics in the Study of CPC Specification}

Accurate quantitative evaluation of genome-wide gene expression provides an essential platform to understand the states of cells of interest. In the case where a specific subset of rare cells plays a vital role, single-cell transcriptomics are a strong tool. Indeed, thus far, this strategy has given fruitful results in the study of the specification of the olfactory receptors, spinal cord neurons and primordial germ cells [11-13]. Currently, several protocols of single-cell transcriptomics have been developed as applicable to next-generation sequencing (NGS) with sufficient coverage, sensitivity and accuracy (Table. 10.1) [2].

The protocol for genome-wide analysis was first developed by Kurimoto and colleagues for microarray and has been recently modified for NGS (Fig. 10.2a) [1416]. The feature of this protocol is to add artificially a poly A tail to the 3 -end of the first-strand cDNA to generate the appropriate adapter sites for PCR amplification. At the final step, cDNA is obtained as PCR amplicon. Disadvantageously Kurimoto's protocol provides only strongly 3'-biased cDNA, although Tang's protocol likely overcomes this problem [15].

Quartz-seq is also a method applicable to microarray and NGS, based on PCR amplification, and the architecture of the protocol is based on "suppressive PCR" (Fig. 10.2a) [17]. This method looks superior to Kurimoto's in terms of simple operation, coverage and technical reproducibility.

SMART-seq utilizes a unique nature of reverse transcriptase [18]. During reverse transcription, Moloney murine leukaemia virus reverse transcriptase adds a short poly $\mathrm{C}$ tail to the $3^{\prime}$-end of the first-strand cDNA. Reverse transcriptase uses a helper oligonucleotide with poly $\mathrm{G}$ motif as the template ("template switch") after annealing to the poly $\mathrm{C}$ tail, which results in the addition of adaptors to cDNA (Fig. 10.2b). This template switch happens preferentially to $5^{\prime}$-capped mRNA so that finally a full-length cDNA library is obtained as suppressive PCR amplicon if oligo(dT) primer is used for the first-strand synthesis. This enrichment of full length

Table 10.1 Methods of single-cell transcriptomics

\begin{tabular}{l|l|l|l}
\hline Method & Principle & Application & References \\
\hline Kurimoto et al. 2006 & Poly A tailing/PCR & Microarray & {$[14]$} \\
\hline Tang et al. 2009 & Poly A tailing/PCR & NGS & {$[15]$} \\
\hline Brouilette et al. 2012 & Poly A tailing/PCR & NGS & {$[16]$} \\
\hline Quartz-seq/-chip & Poly A tailing/suppressive PCR & NGS/microarray & {$[17]$} \\
\hline SMART-seq & Template switch/PCR & NGS & {$[18]$} \\
\hline CEL-seq & IVT/PCR & NGS & {$[19]$} \\
\hline
\end{tabular}


(a)

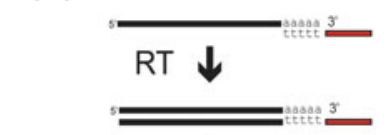

poly A tailing $\downarrow$
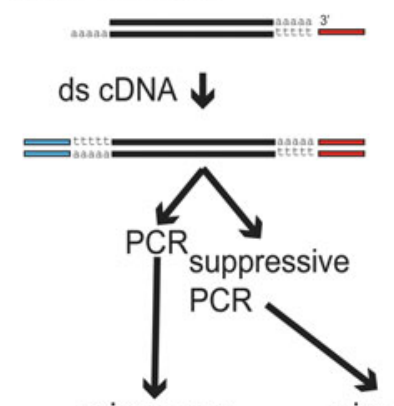

microarray

(Kurimoto's)

NGS (b)
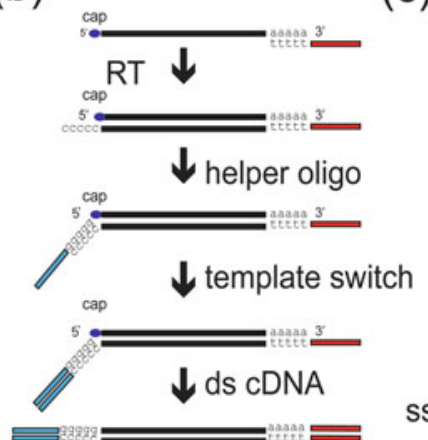

(c)

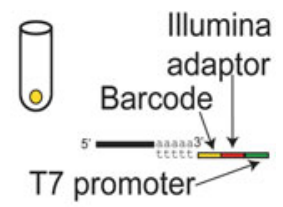

T7 promoter-

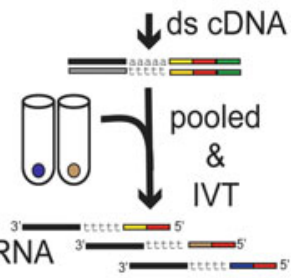

frag

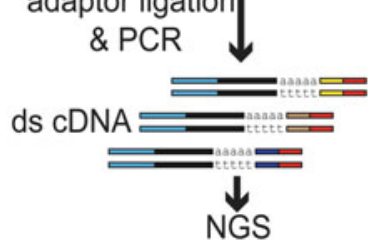

(Tang's \& Brouilette's) (Quartz-seq)

Fig. 10.2 Overview of the methods of single-cell transcriptomics. (a) Poly A tailing (Kurimoto's, Tang's, Boulette's protocol and Quartz-seq), (b) SMART-seq, (c) CEL-seq. Please refer to the main text and the relevant references for details

is a merit, but simultaneously a demerit, because any partially synthesized cDNAs are not amplified so that the yield of cDNAs is inevitably limited.

On the other hand, CEL-seq uses mainly isothermal amplification of in vitro transcription (IVT) by T7 polymerase (Fig. 10.2c) [19]. Theoretically, IVT is superior to PCR in terms of liner and proportional amplification to secure better technical reproducibility. A demerit of this protocol is that reads by NGS are strongly biased toward $3^{\prime}$-end.

Lastly, we should discuss the limitations of single-cell transcriptomics. First, there is always a technical noise due to pippetting error, difference of system (including enzymes, thermal cycler and NGS machine) and amplification bias. Of note, the "Monte Carlo effect", the cause of large quantitative errors of small copy number mRNAs due to the stochastic events within an initial few cycles of PCR reaction, is uncontrollable. Amplification bias is inevitable to some extent in the protocols dependent on PCR, although every protocol has been well validated to secure its sufficient technical reproducibility. Second, the lack of topological information precludes us from referring the obtained expression profile to the cell in the embryo if no molecular markers exist. Thus it is necessary to consider how to fill such lost information. 


\subsection{Future Direction and Clinical Implication}

The strategy of single-cell transcriptomics has great potential to increase understanding of CPC specification, their ground state and differentiation. This knowledge will be utilized in order to control the commitment of pluripotent stem cells including ES cells and iPS cells for future clinical application as stem cell-based regeneration therapy.

Acknowledgements We thank Kazuko Koshiba-Takeuchi, Jun Takeuchi, Shigetoyo Kogaki, Manabu Shirai, Hiroki Kokubo, Yusuke Watanabe and Hiroyuki Yamagishi for helpful discussions and Steven Coppen for editing. Our work is supported by MRC New Investigator Research Grant G0900105, MRC Research Grant MR/J007625/1 and BHF Project Grant PG/11/ $102 / 29213$ to $\mathrm{KY}$.

Open Access This chapter is distributed under the terms of the Creative Commons AttributionNoncommercial 2.5 License (http://creativecommons.org/licenses/by-nc/2.5/) which permits any noncommercial use, distribution, and reproduction in any medium, provided the original author(s) and source are credited.

The images or other third party material in this chapter are included in the work's Creative Commons license, unless indicated otherwise in the credit line; if such material is not included in the work's Creative Commons license and the respective action is not permitted by statutory regulation, users will need to obtain permission from the license holder to duplicate, adapt or reproduce the material.

\section{References}

1. Rana MS, Christoffels VM, Moorman AF. A molecular and genetic outline of cardiac morphogenesis. Acta Physiol (Oxf). 2013;207:588-615.

2. Shapiro E, Biezuner T, Linnarsson S. Single-cell sequencing-based technologies will revolutionize whole-organism science. Nat Rev Genet. 2013;14:618-30.

3. Kinder SJ, Tsang TE, Quinlan GA, et al. The orderly allocation of mesodermal cells to the extraembryonic structures and the anteroposterior axis during gastrulation of the mouse embryo. Development. 1999;126:4691-701.

4. Tam PP, Parameswaran M, Kinder SJ, et al. The allocation of epiblast cells to the embryonic heart and other mesodermal lineages: the role of ingression and tissue movement during gastrulation. Development. 1997;124:1631-42.

5. Takeuchi JK, Bruneau BG. Directed transdifferentiation of mouse mesoderm to heart tissue by defined factors. Nature. 2009;459:708-11.

6. Cai CL, Liang X, Shi Y, et al. Isl1 identifies a cardiac progenitor population that proliferates prior to differentiation and contributes a majority of cells to the heart. Dev Cell. 2003;5:877-89.

7. Moretti A, Caron L, Nakano A, et al. Multipotent embryonic isl1+ progenitor cells lead to cardiac, smooth muscle, and endothelial cell diversification. Cell. 2006;127:1151-65.

8. Prall OW, Menon MK, Solloway MJ, et al. An Nkx2-5/Bmp2/Smad1 negative feedback loop controls heart progenitor specification and proliferation. Cell. 2007;128:947-59.

9. Ma Q, Zhou B, Pu WT. Reassessment of Is11 and Nkx2-5 cardiac fate maps using a Gata4based reporter of Cre activity. Dev Biol. 2008;323:98-104.

10. Nemir M, Croquelois A, Pedrazzini T, et al. Induction of cardiogenesis in embryonic stem cells via downregulation of Notch1 signaling. Circ Res. 2006;98:1471-8. 
11. Dulac C, Axel R. A novel family of genes encoding putative pheromone receptors in mammals. Cell. 1995;83:195-206.

12. Tanabe Y, William C, Jessell TM. Specification of motor neuron identity by the MNR2 homeodomain protein. Cell. 1998;95:67-80.

13. Saitou M, Barton SC, Surani MA. A molecular programme for the specification of germ cell fate in mice. Nature. 2002;418:293-300.

14. Kurimoto K, Yabuta Y, Ohinata Y, et al. An improved single-cell cDNA amplification method for efficient high-density oligonucleotide microarray analysis. Nucleic Acids Res. 2006;34: e42.

15. Tang F, Barbacioru C, Wang Y, et al. mRNA-Seq whole-transcriptome analysis of a single cell. Nat Methods. 2009;6:377-82.

16. Brouilette S, Kuersten S, Mein C, et al. A simple and novel method for RNA-seq library preparation of single cell cDNA analysis by hyperactive Tn5 transposase. Dev Dyn. 2012;241:1584-90.

17. Sasagawa Y, Nikaido I, Hayashi T, et al. Quartz-Seq: a highly reproducible and sensitive single-cell RNA sequencing method, reveals non-genetic gene-expression heterogeneity. Genome Biol. 2013;14:R31.

18. Ramskold D, Luo S, Wang YC, et al. Full-length mRNA-Seq from single-cell levels of RNA and individual circulating tumor cells. Nat Biotechnol. 2012;30:777-82.

19. Hashimshony T, Wagner F, Sher N, et al. CEL-Seq: single-cell RNA-Seq by multiplexed linear amplification. Cell Rep. 2012;2:666-73. 